\title{
Effects of laterality and pitch height of an auditory accessory stimulus on horizontal response selection: The Simon effect and the SMARC effect
}

\author{
AKIO NiShimura AND KazUhiko YokosaWa \\ University of Tokyo, Tokyo, Japan
}

\begin{abstract}
In the present article, we investigated the effects of pitch height and the presented ear (laterality) of an auditory stimulus, irrelevant to the ongoing visual task, on horizontal response selection. Performance was better when the response and the stimulated ear spatially corresponded (Simon effect), and when the spatial-musical association of response codes (SMARC) correspondence was maintained - that is, right (left) response with a high-pitched (low-pitched) tone. These findings reveal an automatic activation of spatially and musically associated responses by task-irrelevant auditory accessory stimuli. Pitch height is strong enough to influence the horizontal responses despite modality differences with task target.
\end{abstract}

Performance is better when a stimulus and a response share some features than when they do not, regardless of the task relevancy of the shared feature (Kornblum, Hasbroucq, \& Osman, 1990; Proctor \& Vu, 2006). For example, right responses to stimuli presented to the right side are faster than those presented to the left side, whereas the reverse pattern holds with the left responses, resulting in the stimulus-response compatibility (SRC) effect. Similar compatibility effects are observed not only when participants respond to stimulus location, but also when they respond on the basis of some nonspatial feature of the stimulus, such as color or shape; this phenomenon has been labeled the Simon effect (see Lu \& Proctor, 1995, for a review). In a typical Simon task, participants respond with a right or left keypress to the color of the target stimulus that randomly appears at a right or left position.

The Simon effect is not restricted to the spatial information associated with a target stimulus in some task. Rather, it emerges even when the spatial information is associated with accompanying, task-irrelevant (i.e., termed accessory) visual or auditory stimuli-namely, stimuli that differ from the target. In a typical accessory Simon task, participants engage in a task that requires responses to a central visual target using response keys arranged horizontally. In this case, responses are faster when a task-irrelevant visual or auditory accessory stimulus is presented on the same side as the appropriate response key than when it is presented on the side opposite to this response key (see, e.g., Proctor, Pick, Vu, $\&$ Anderson, 2005). Such results indicate that irrelevant visual or auditory stimuli are strong enough to activate a spatial congruent response in spite of the fact that they are irrelevant to the task and may even involve a different modality.

Stimulus features other than horizontal spatial features are also known to affect horizontal (i.e., right and left) response selection. For example, with vertical stimulus arrays and horizontal arrangements of response keys, performance is better for stimulus-response mappings that combine higher (up) stimuli with right response keys and lower (down) stimuli with left response keys - that is, up-right/ down-left - than for the opposite mappings (Cho \& Proctor, 2003; Weeks \& Proctor, 1990). This orthogonal SRC effect also emerges when the location of a visual stimulus is task irrelevant (Nishimura \& Yokosawa, 2006). Numerical magnitude also systematically affects horizontal responses. Right responses are facilitated by stimuli that reflect smaller numbers, whereas left responses are facilitated by stimuli of larger numbers; this is known as the spatial-numerical association of response codes (SNARC) effect (Dehaene, Bossini, \& Giraux, 1993). Parity also affects horizontal responses, with better performance for odd-left/even-right stimulus-response mapping than for even-left/odd-right mapping; this is known as the linguistic markedness association of response codes (MARC) effect (Nuerk, Iversen, \& Willmes, 2004; Reynvoet \& Brysbaert, 1999). Gevers, Reynvoet, and Fias (2003) showed that various ordinal sequences, such as alphabet and months, also systematically affect horizontal responses. Beginning (ending) elements of an ordinal sequence facilitated left (right) responses, and ordinal effects emerged even when the order was irrelevant to the task. In addition, some temporal factors have been reported to differentially influence horizontal responses. Santiago, Lupiáñez, Pérez, and Funes (2007) found a past-

K.Yokosawa, yokosawa@l.u-tokyo.ac.jp 
left/future-right association in a task requiring participants to press the right or left key to categorize words as referring to the past or to the future, respectively. Ishihara, Keller, Rossetti, and Prinz (2008) found that the left responses are faster to stimuli that occur unexpectedly early in time, whereas right responses are faster to stimuli occurring unexpectedly late. Ishihara et al. termed this time-space association the spatial-temporal association of response codes (STEARC) effect. Thus, many stimulus factors have been shown to influence the horizontal responses, which indicates that the influence of perceptual factors on right and left responses may be a universal phenomenon and that studies of these effects are important to understanding interactions of perception and action.

Recently, Rusconi, Kwan, Giordano, Umiltà, and Butterworth (2006) and Lidji, Kolinsky, Lochy, and Morais (2007) reported an effect of auditory pitch on differential responding to spatially distinct response keys. In Rusconi et al., on each trial, following the fixed reference tone, a tone of higher or lower frequency was presented through headphones; nonmusicians had to judge whether the pitch of the second tone was higher or lower than the referent pitch. With horizontally arranged keys, these subjects showed a tendency toward associating the higher (lower) pitched stimuli with the right (left) response; that is, they were faster with the stimulus-response mapping of lowerleft/higher-right than with the mapping of lower-right/ higher-left. This effect has been labeled the spatialmusical association of response codes (SMARC) effect. Although the SMARC effect was absent when nonmusicians engaged in a musical instrument identification task in which the pitch height was task irrelevant, it was present for musicians in this task. Lidji et al. also reported a SMARC effect based on the pitch height of an isolated tone with a pitch comparison task in nonmusicians, and with an instrument identification task only in musicians. Thus, the relative pitch height of auditory stimuli is closely associated with the right and left responses.

Previous studies have shown that the pitch of an auditory stimulus can selectively activate either a right or left response, even when it is irrelevant to the task, at least for musicians. However, although pitch was irrelevant in these tasks, the target itself was an auditory stimulus; hence, irrelevant and relevant features shared the same modality. Therefore, it is possible that the SMARC effect in those studies was due to incorporating incidental pitch information into the perceptual representation of the auditory target stimulus. On the other hand, studies of the Simon effect have repeatedly reported a systematic impact of visual or auditory spatial accessory stimuli that differ from the target of the task. This finding indicates that an irrelevant stimulus feature that is different from the target modality can influence horizontal responses. In the present study, we investigated the influence of a nonspatial feature of taskirrelevant auditory stimuli on the horizontal responses to visual stimuli. Participants engaged in a visual task in which they responded to a centrally presented visual stimulus with right or left keypresses; simultaneously with the presentation of a visual target, participants heard an accessory auditory stimulus (low or high pure tone) monaurally through headphones either to their right or left ear. For simplicity, we used only two pitch heights; thus, the two tones can be defined relative to each other as high- and low-pitched tones.

The main purpose of the present study was to investigate the automaticity of the effect of relative pitch height on the horizontal responses. Therefore, we posed the following question: Does relative pitch height - although irrelevant to the target and the task (and, indeed, associated with an irrelevant modality) - affect horizontal response selection in a visual task in the same (automatic) fashion as does manipulation of the spatial location of taskirrelevant sound sources (e.g., as in Proctor et al., 2005)? If pitch height is strong enough to elicit the right or left response, then the SMARC effect would be obtained even in the present task setting. If the SMARC effect in previous studies was the result of the task setting in which pitch features of the auditory stimulus were influential because of general attention to the auditory target, then the SMARC effect would not be obtained in the present task setting. In addition to probing for a SMARC effect, we expected to find an accessory Simon effect, as in the previous studies. Finally, in our paradigm, we could also evaluate contributions from another potential influence on responding - namely, the S-S congruency. An association of the stimulus features - pitch height and tone location (laterality) - may affect the performance: It is possible that performance will be better when the high tone is presented to the right ear and when the low tone is presented to the left ear than when an opposite combination of pitch height and presented ear obtains.

\section{METHOD}

\section{Participants}

Sixteen volunteers ${ }^{1}$ ( 9 males; mean age $=20.9$ years; 15 righthanded) participated in this experiment. All had normal or correctedto-normal vision and reported no hearing problems.

\section{Apparatus and Stimuli}

Stimulus presentation and response recording were controlled by an AV-tachistoscope system (Iwatsu ISEL IS-703). Visual stimuli were presented on a 22-in. monitor. The response apparatus consisted of right and left keys that were approximately $7 \mathrm{~cm}$ apart from each other and approximately $21 \mathrm{~cm}$ in front of the participants. A head- and chinrest ensured that participants maintained a viewing distance of $57 \mathrm{~cm}$. Auditory stimuli were presented monaurally through headphones (AKG K271 studio).

The visual stimulus was a central cross $(5 \times 5 \mathrm{~mm})$ presented on a black background. Two auditory accessory stimuli involved a lowpitched pure tone $(300 \mathrm{~Hz})$ and a high-pitched pure tone $(500 \mathrm{~Hz})$. The intensity of each stimulus was $76 \mathrm{~dB}$. The duration of each tone was 2,000 msec (including a linear amplitude ramp of $10 \mathrm{msec}$ ).

\section{Task and Procedure}

The participants' task was to press the right or left response key according to the color of the visual stimulus as quickly as possible while maintaining high accuracy. Half of the participants responded to a green target stimulus with a right keypress and to a red target stimulus with a left keypress using the index fingers of the corresponding hand. The color-to-key mapping was reversed with the remaining half. The auditory "accessory" stimuli were irrelevant to the task.

Each trial was initiated by the presentation of the white fixation cross in the center of the screen for 2,000 msec. Then, the fixation cross turned red or green. Simultaneously with the color change of 
the visual stimulus, a high- or low-pitched tone was presented to the left or right ear through headphones. After 2,000 msec, both the visual and the auditory stimuli disappeared. The next trial started after the intertrial interval of $1,000 \mathrm{msec}$. Participants engaged in three experimental blocks of 80 trials each; these were preceded by a practice block of 16 trials. Short rest breaks were provided between trial blocks. Each experimental block consisted of 10 replications for each type, defined by a factorial combination of stimulus color (green, red), pitch height $(300 \mathrm{~Hz}, 500 \mathrm{~Hz})$, and tone laterality (left, right), and the trials were presented in a random order.

\section{RESULTS}

We restricted analyses to the 15 right-handers because the interaction between pitch height and horizontal spatial location is known to be affected by handedness (see, e.g., Deutsch, 1995). ${ }^{2}$ Trials on which participants failed to respond, as well as trials on which reaction times (RTs) exceeded 1,000 msec or were less than $200 \mathrm{msec}$, were excluded from the data analyses $(0.25 \%$ of all the trials $)$. Mean RTs for correct responses and the percentages of errors were submitted to separate ANOVAs with tone laterality (left, right), pitch height (low, high), and response (left, right) as within-participants factors.

\section{RT}

The two-way interaction between tone laterality and response was significant $\left[F(1,14)=28.06, M S_{\mathrm{e}}=259.73\right.$, $p<.001]$, indicating an accessory Simon effect. Responses were faster when tone laterality and the requisite response were in agreement-that is, on the same side ( $M=$ $378 \mathrm{msec})$ - than when they were on opposite sides $(M=$ $394 \mathrm{msec}$ ), indicative of a Simon effect. The two-way interaction between pitch height and response was also significant $\left[F(1,14)=5.67, M S_{\mathrm{e}}=273.37, p<.05\right]$, indicating a SMARC effect. Responses were faster for low pitch-left responses and for high pitch-right responses $(M=382 \mathrm{msec})$ than for low pitch-right responses and high pitch-left responses $(M=390 \mathrm{msec})$. Other main effects or interactions were not significant $(p \mathrm{~s}>.29)$. Figure 1 plots the RT differences captured by both of these interactions as a function of the type of compatibility (Simon, SMARC).

To reveal the time course of each of the two observed effects (auditory accessory Simon effect and SMARC effect), we conducted distribution analyses of these RTs

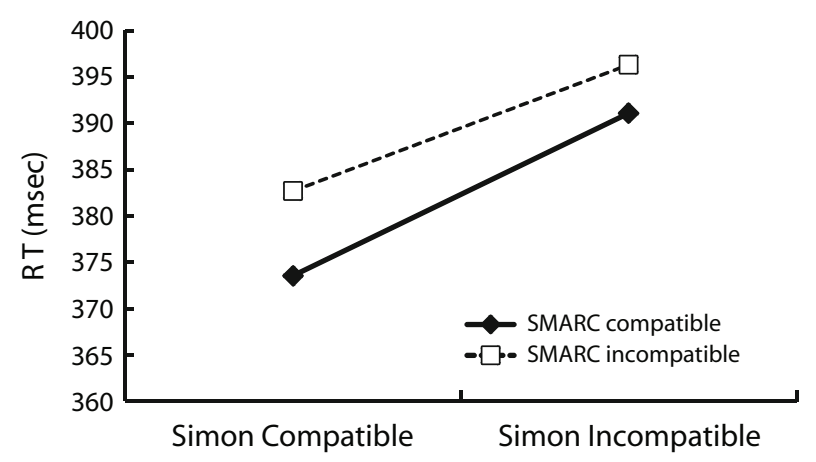

Figure 1. Mean reaction times (RTs, in milliseconds) as a function of Simon compatibility and SMARC compatibility.
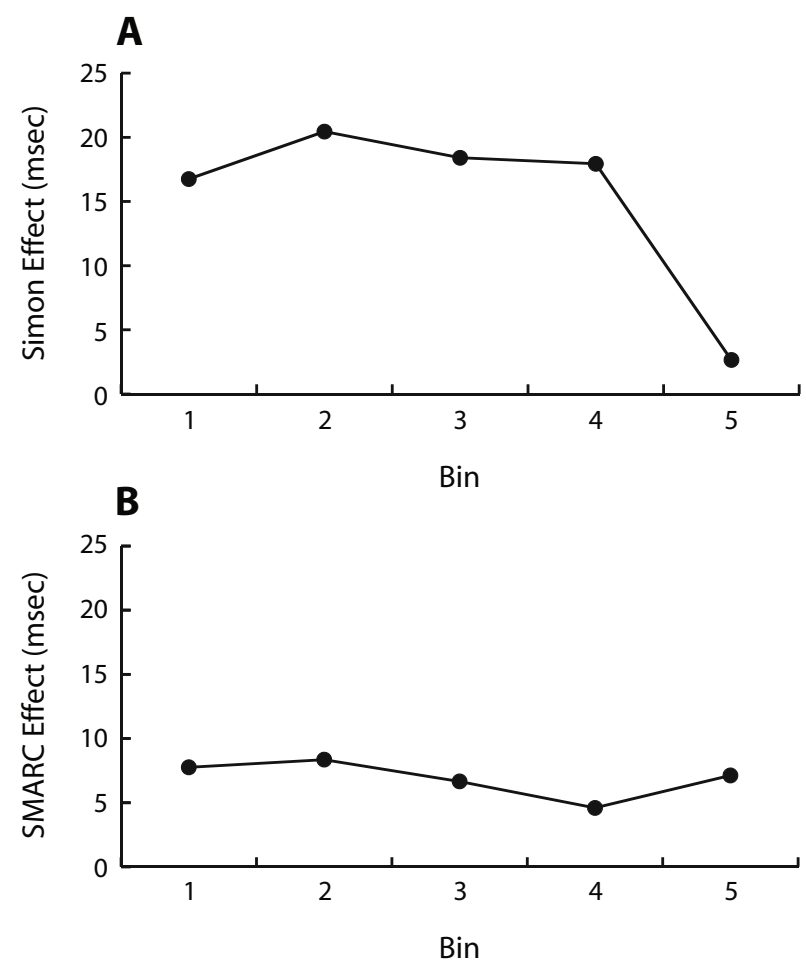

Figure 2. The Simon effect and SMARC effect (reaction time difference between imcompatible and compatible conditions) as a function of bin.

(De Jong, Liang, \& Lauber, 1994; Ratcliff, 1979). Separate analyses were conducted on the Simon effect and the SMARC effect. For these analyses, correct RTs over all trials for each participant were rank ordered from the fastest to the slowest for each of the two compatibility conditions (compatible, incompatible). Each RT distribution was divided into quintile bins. The mean RT for each bin was submitted to an ANOVA with compatibility and bin as within-participants factors. For the Simon effect, the main effects of both compatibility $[F(1,14)=26.84$, $\left.M S_{\mathrm{e}}=324.32, p<.001\right]$ and bin $[F(4,56)=129.30$, $\left.M S_{\mathrm{e}}=1,085.36, p<.001\right]$ were significant. Most importantly, the two-way interaction was significant $\left[F(4,56)=4.82, M S_{\mathrm{e}}=79.84, p<.005\right]$. The Simon effect was observed for the first four bins $(p s<.001)$, but was absent for the slowest bin $(p>.99)$ (Figure 2A). For the SMARC effect, the main effects of both compatibility $\left[F(1,14)=5.28, M S_{\mathrm{e}}=337.79, p<.05\right]$ and bin $\left[F(4,56)=134.73, M S_{\mathrm{e}}=1,064.10, p<.001\right]$ were significant. However, the two-way interaction was far from significant $(F<1)$. The SMARC effect was constant across the RT bins (Figure 2B).

\section{Error Rate}

The two-way interaction between tone laterality and response was significant $\left[F(1,14)=8.42, M S_{\mathrm{e}}=13.65\right.$, $p<.05]$, indicating an accessory Simon effect. Participants responded more accurately when tone laterality and the response were on the same side $(1.7 \%)$ than on the opposite sides $(3.6 \%)$. Other main effects or interactions 
were not significant $(p \mathrm{~s}>.09)$. No significant SMARC effect was observed in error rate $(0.5 \%$; pitch height $\times$ response, $F<1$ ).

\section{DISCUSSION}

The accessory Simon effect and the accessory SMARC effect were obtained simultaneously in the present experiment. Performance was better when the right responses were accompanied by the presentation of a right lateralized tone and when the left responses were accompanied by the presentation of a left lateralized tone than it was when the reverse combinations were presented. In addition, an accessory SMARC effect was revealed with better performance when the right responses were accompanied by the high-pitched tone and the left responses were accompanied by the low-pitched tone than when the right responses were accompanied by the low-pitched tone and the left responses were accompanied by the high-pitched tone. Thus, the present study revealed that both laterality and pitch height of a presented tone can automatically activate congruent responses, even when the tone is an accessory stimulus that is different from the task target and when the auditory modality is task irrelevant. This result indicates that the SMARC effect in previous studies (Lidji et al., 2007; Rusconi et al., 2006) was not due to the task settings and that pitch height of auditory stimulus is strong enough to influence the horizontal responses over the target modality of the task.

In contrast with the significant two S-R effects, S-S congruency effect was not observed in the present experiment. The two-way interaction between tone laterality and the pitch height was not significant for either RT ( $p=.33$ ) or error rate $(p=.09)$. Moreover, the $\mathrm{S}-\mathrm{S}$ congruency effect trended in the opposite directions for RT $(2 \mathrm{msec})$ and error rate $(-0.7 \%)$. Although pitch height influences its representation in cognitive space (see, e.g., Mudd, 1963), the combination of pitch height and tone laterality did not influence speeded horizontal responses on the basis of a visual target.

Wiegand and Wascher (2007) have assumed that two types of the Simon effects exist and that they are based on distinct underlying mechanisms. One is a visuomotor Simon effect, which depends on a transmission of visuomotor information and is characterized by the decreasing effect function over time. The other Simon effect is cognitive in nature and is assumed to derive from cognitive code interference; it is characterized by stable or increasing effect function over time. In the present experiment, the two-way interaction between bin and compatibility was significant for the Simon effect but not for the SMARC effect. The accessory SMARC effect was constant across RT bins. The auditory accessory Simon effect disappeared at the fifth bin, whereas it was constant across the first four bins. The effect function was not precisely decreasing (see Figure 2). In fact, for an auditory Simon effect, previous studies have reported a stable or an increasing effect function, thereby suggesting the presence of cognitive code interference (see, e.g., Proctor et al., 2005; Wascher, Schatz, Kuder, \& Verleger, 2001). Although both the ac- cessory Simon effect and the SMARC effect may be cognitive in nature, they appear to have exhibited different time courses in the present experiment. The Simon effect and the SMARC effect did not interact. The three-way interaction did not even approach significance in either the $\mathrm{RT}(p=.82)$ or error rate $(p=.75)$ analysis. Although additive factors logic (Sternberg, 1969) predicts an interaction when two effects emerge within the same processing stage, it should be noted that an absence of the significant interaction (in the present case) does not guarantee that the two effects necessarily emerge at separate stages. One interpretation of our observation of different time courses of Simon versus SMARC effects (along with possible additivity of these effects) is that different mechanisms may be associated with them. However, this interpretation is tentative and should not be viewed as conclusive.

In previous SMARC studies (Lidji et al., 2007; Rusconi et al., 2006), the SMARC effect was obtained only with musically experienced participants when the pitch height was irrelevant to the task. In the present study, all of the participants had experience with musical training of approximately $1.3 \mathrm{~h}$ per week during 9 years of elementary and junior high school compulsory education. Except for 1 participant, they had additional music experience of 2 to 15 years, partially in parallel with the compulsory education. However, the amount of additional music experience did not correlate with the size of the SMARC effect $(r=$ .02 for RT and $r=.07$ for error rate). The musical experience during elementary and junior high school might have been sufficient to form the spatial-musical association that elicits the SMARC effect. Moreover, we used only two pitches in the present study. As a result, each tone was uniquely defined as high or low in relation to the other tone. Using just two tones may also facilitate the SMARC effect for musically less experienced participants.

The present study revealed that the laterality and the pitch height of a presented tone can simultaneously affect the right and left responses, even when the tone is irrelevant to the task. In addition to tone pitch, vertical stimulus location (Cho \& Proctor, 2003; Nishimura \& Yokosawa, 2006), number (Dehaene et al., 1993; Nuerk et al., 2004), member of ordinal sequences (Gevers et al., 2003), and time (Ishihara et al., 2008; Santiago et al., 2007) are all known to differentially affect the selection of horizontally arranged response keys. As was pointed out by Rusconi et al. (2006) and Lidji et al. (2007), the SMARC effect may be a variant of the orthogonal compatibility effect (i.e., up-right/down-left advantage). Pitch height is closely related to vertical spatial location (e.g., Melara \& O'Brien, 1987). Thus, the orthogonal compatibility effect and the SMARC effect may reflect explicit and implicit vertical spatial properties of a stimulus. The SMARC effect's being smaller than the Simon effect in the present study is consistent with the size difference between the orthogonal and the parallel compatibility effects (see Nishimura \& Yokosawa, 2006). In addition, different time courses for Simon and orthogonal SRC effects, with the latter being constant over time, were reported in Cho and Proctor (2005). A similar dissociation of time course also appears to hold for Simon and SNARC effects (see, 
e.g., Mapelli, Rusconi, \& Umiltà, 2003). These effects of nonhorizontal stimulus features on horizontal responses may be explained within a single theoretical framework (see Proctor \& Cho, 2006), at least to a large extent. Future research concerning mechanisms responsible for commonalities as well as differences underlying these effects will advance our understanding of basic aspects of the interaction between perception and action.

\section{AUTHOR NOTE}

The present study was supported by Grant 18-10669 from the Research Fellowships of the Japan Society for the Promotion of Science for Young Scientists to A.N., and by Grant-in-Aid for Scientific Research 17330152 from the Japan Society for the Promotion of Science awarded to K.Y. A.N. is now at Tohoku University. Correspondence concerning this article should be addressed to K. Yokosawa, Department of Psychology, Graduate School of Humanities and Sociology, University of Tokyo, 7-3-1 Hongo, Bunkyo-ku, Tokyo 113-0033, Japan (e-mail: yokosawa@1.u-tokyo.ac.jp).

\section{REFERENCES}

Chо, Y. S., \& Proctor, R. W. (2003). Stimulus and response representations underlying orthogonal stimulus-response compatibility effects. Psychonomic Bulletin \& Review, 10, 45-73.

Сно, Y. S., \& Рвостов, R. W. (2005). Representing response position relative to display location: Influence on orthogonal stimulusresponse compatibility. Quarterly Journal of Experimental Psychology, 58A, 839-864. doi:10.1080/02724980443000359

Dehaene, S., Bossini, S., \& Giraux, P. (1993). The mental representation of parity and number magnitude. Journal of Experimental Psychology: General, 122, 371-396.

De Jong, R., Liang, C.-C., \& Lauber, E. (1994). Conditional and unconditional automaticity: A dual-process model of effects of spatial stimulus-response correspondence. Journal of Experimental Psychology: Human Perception \& Performance, 20, 731-750.

Deutsch, D. (1995). Musical illusions and paradoxes. La Jolla, CA: Philomel Records.

Gevers, W., Reynvoet, B., \& Fias, W. (2003). The mental representation of ordinal sequences is spatially organized. Cognition, 87, B87B95. doi:10.1016/S0010-0277(02)00234-2

Ishihara, M., Keller, P. E., Rossetti, Y., \& Prinz, W. (2008). Horizontal spatial representations of time: Evidence for the STEARC effect. Cortex, 44, 454-461. doi:10.1016/j.cortex.2007.08.010

Kornblum, S., Hasbrouce, T., \& Osman, A. (1990). Dimensional overlap: Cognitive basis for stimulus-response compatibility - A model and taxonomy. Psychological Review, 97, 253-270.

LidjI, P., Kolinsky, R., Lochy, A., \& Morais, J. (2007). Spatial associations for musical stimuli: A piano in the head? Journal of Experimental Psychology: Human Perception \& Performance, 33, 1189-1207. doi:10.1037/0096-1523.33.5.1189

Lu, C.-H., \& Proctor, R. W. (1995). The influence of irrelevant location information on performance: A review of the Simon and spatial Stroop effects. Psychonomic Bulletin \& Review, 2, 174-207.

Mapelli, D., Rusconi, E., \& Umiltà, C. (2003). The SNARC effect: An instance of the Simon effect? Cognition, 88, B1-B10. doi:10.1016/ S0010-0277(03)00042-8
Melara, R. D., \& O'Brien, T. P. (1987). Interaction between synesthetically corresponding dimensions. Journal of Experimental Psychology: General, 116, 323-336.

MudD, S. A. (1963). Spatial stereotypes of four dimensions of pure tone. Journal of Experimental Psychology, 66, 347-352.

Nishimura, A., \& YoKosaWa, K. (2006). Orthogonal stimulus-response compatibility effects emerge even when the stimulus position is task irrelevant. Quarterly Journal of Experimental Psychology, 59, 10211032. doi: $10.1080 / 17470210500416243$

Nuerk, H.-C., Iversen, W., \& Willmes, K. (2004). Notational modulation of SNARC and the MARC (linguistic markedness of response codes) effect. Quarterly Journal of Experimental Psychology, 57A, 835-863. doi:10.1080/02724980343000512

Proctor, R. W., \& CHo, Y. S. (2006). Polarity correspondence: A general principle for performance of speeded binary classification tasks. Psychological Bulletin, 132, 416-442. doi:10.1037/0033 $-2909.132 .3 .416$

Proctor, R. W., Pick, D. F., Vu, K.-P. L., \& Anderson, R. E. (2005). The enhanced Simon effect for older adults is reduced when the irrelevant location information is conveyed by an accessory stimulus. Acta Psychologica, 119, 21-40. doi:10.1016/j.actpsy.2004.10.014

Proctor, R. W., \& Vu, K.-P. L. (2006). Stimulus-response compatibility principles: Data, theory, and application. Boca Raton, FL: CRC Press.

RATCLIFF, R. (1979). Group reaction time distributions and an analysis of distribution statistics. Psychological Bulletin, 86, 446-461.

Reynvoet, B., \& Brysbaert, M. (1999). Single-digit and two-digit Arabic numerals address the same semantic number line. Cognition, 72, 191-201

Rusconi, E., Kwan, B., Giordano, B. L., Umiltà, C., \& ButterWORTH, B. (2006). Spatial representation of pitch height: The SMARC effect. Cognition, 99, 113-129. doi:10.1016/j.cognition.2005.01.004

Santiago, J., Lupiáñez, J., Pérez, E., \& Funes, M. J. (2007). Time (also) flies from left to right. Psychonomic Bulletin \& Review, 14, 512-516.

STERNBERG, S. (1969). The discovery of processing stages: Extensions of Donders' method. Acta Psychologica, 30, 276-315.

Wascher, E., Schatz, U., Kuder, T., \& Verleger, R. (2001). Validity and boundary conditions of automatic response activation in the Simon task. Journal of Experimental Psychology: Human Perception \& Performance, 27, 731-751.

Weeks, D. J., \& Proctor, R. W. (1990). Salient-features coding in the translation between orthogonal stimulus and response dimensions. Journal of Experimental Psychology: General, 119, 355-366.

Wiegand, K., \& Wascher, E. (2007). The Simon effect for vertical S-R relations: Changing the mechanism by randomly varying the S-R mapping rule? Psychological Research, 71, 219-233. doi:10.1007/ s00426-005-0023-5

\section{NOTES}

1. We discuss the participants' musical experience later in the Discussion section.

2. We thank Trammell Neill for advising us to analyze data in this way.

(Manuscript received May 23, 2008;

revision accepted for publication February 22, 2009.) 